\title{
Is agro-tourism eco-friendly?
}

\author{
Ramona Giurea ${ }^{1, *}$, Marco Ragazzi ${ }^{2}$, Moise Ioan Achim ${ }^{3}$, and Mihai-Victor Zerbes ${ }^{4}$ \\ ${ }^{1}$ Department Engineering and Management, Lucian Blaga University of Sibiu, 550024, Sibiu, \\ Romania \& Department of Civil Environmental and Mechanical Engineering, University of Trento, \\ 38123, Trento, Italy \\ ${ }^{2}$ Department of Civil Environmental and Mechanical Engineering, University of Trento, 38123 , \\ Trento, Italy \\ ${ }^{3}$ Department of Exact Sciences and Engineering, "1 Decembrie 1918” University of Alba Iulia, \\ 510009, Alba Iulia, Romania \\ ${ }^{4}$ Department Engineering and Management, Lucian Blaga University of Sibiu, 550024, Sibiu, \\ Romania
}

\begin{abstract}
The paper will highlight the impact of agro-tourism on environment in Romania, focusing on the Sibiu County. Thanks to the results obtained from this analysis, research and information collection, the paper will give some answers regarding the question: are agro-tourisms environmentally friendly? The authors identify potential causes of environmental pollution from agro-tourism and some resources that could be economically used. The negative and positive aspects on the increase of agro-tourism activities in the proposed region will be also discussed taking into account there impact on the environment. Based on the obtained results, the authors will propose a method for the management of resources in order to arrive to an eco-friendly system for the proposed case-study. Future developments and applications on other EU case-study will be also discussed in this paper.
\end{abstract}

\section{European framework on environment}

The fundamental of our health, our economy and our well-being is the environmental quality. Nevertheless, it faces several serious challenges as climate change, unsustainable consumption and production, as well as various forms of pollution [1-3]. A part of this pollution is also connected with the agro-tourism production and consumption: wastewater, solid waste, energy consumption, etc. [4,5]

The concern for environmental protection appeared on the European Agenda in the early 1970s [6]. Environmental policy of the European Union (EU) was created by the Treaty of the European Community with the aims to ensure sustainable environmental protection measures. The Treaty of Maastricht (1993) made the environment an official EU policy area [7]. Over the past decades, the EU has put in place a broad range of environmental legislation through Environment Action Programme (EAP) that sets out priority objectives as: the protection of nature, stronger ecological resilience,

\footnotetext{
*Corresponding author: ramona.giurea@ulbsibiu.ro
} 
sustainable, resource-efficient and low-carbon growth; and the fight against environment-related threats to health [8].

In Romania, after the communist period (1989), began a long process of social, political and economic transformation, whose essence lies in the transition from a planned economy to a free market. So by Government Decision the Ministry of the Environment was established - a state controlled authority, which organizes the institutional framework, develop, guide and improves environmental activities on a national scale [9]. The main areas of expertise of Ministry of Environment are: air quality and environmental noise, management of protected areas, environmental infrastructure, waste management, sustainable development, climatic changes, water management, forestry sector management, etc [10].

The European Union has some of the world's highest environmental standards, developed over decades. Environment policy helps the EU economy become more environmentally friendly, protects Europe's natural resources, and safeguards the health and wellbeing of people living in the EU. The EU environment policy is based on Articles 11 and 191-193 of the Treaty on the Functioning of the European Union [11].

Apart from demands required at European and national level, there are a range of international standards adopted by European Union and implicitly of Romania. The principal standard in Romania is Environment ISO 14001 and in Europe EU Eco-Management and Audit Scheme (EMAS) [12-13].

Tourism plays a major role in the EU economy. According to the European Commission, it is the third largest socio-economic activity in the EU [14]. In the scientific literature we can find the term agro-tourism defined as a subset of rural tourism [15], having the roots on the use of the resources from the rural regions [16-17]. However, rural tourism is an intricate and extended phenomenon and the effect is directly proportional with each individual territory and the relationships between tourism products and local resources [18]. Agrotourism includes various tourist activities, such as accommodation (bed and breakfast, rural lodgings, farm campsite); catering (evening meals); leisure activities (pedagogical farms, sports, horse-riding, farm visits) [19].

The existences of an economic interest and social benefits of agro-tourism is increasing [20-21], specific literature has considerable gaps in which concern environmental consequences [22-23], those gaps being a considerable opportunities to improve the specific processes of the field. Starting in the context of environmental actions, based on analysis, mainly case studies, we can observe that the agro-tourism can produce both positive and negative effects on the environment; for example, some positive effects on environment are: water and energy resources, landscape, biodiversity, reduced use of fertilizers and pesticides in productive processes and improvement of the quality of foods [24].

\section{Proposed methodology}

The methodology used in this article by the authors is inspired from the DMAIC model used by organizations in the six-sigma projects [25]. The overall methodology of the study (Fig. 1, Fig. 2) is detailed explained in an article [26], concerning "The modelling of the improving environmental aspects process and of the associated impacts in industrial organizations" which illustrates the key steps carried out in the model building. 


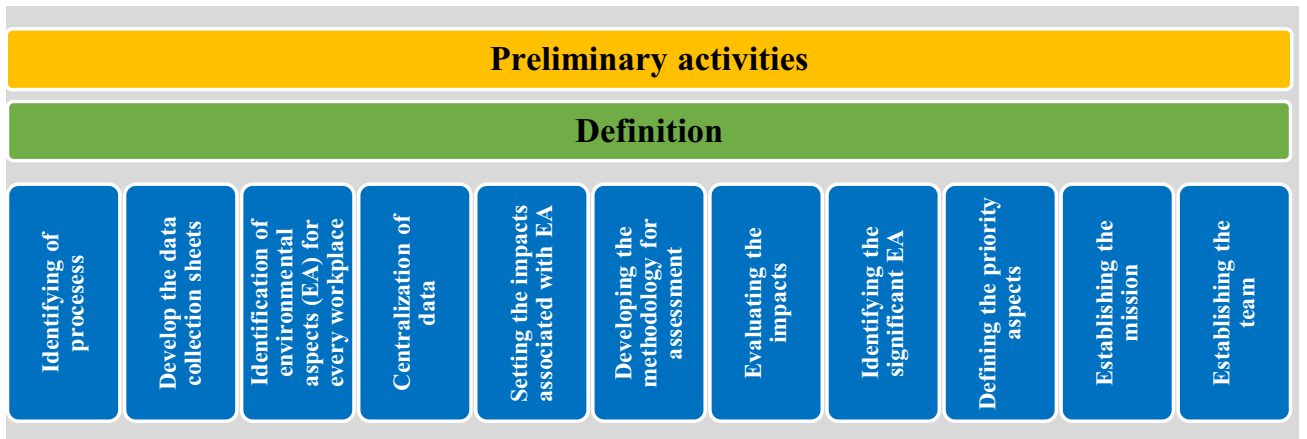

Fig. 1. Phase of the propose DMAIC model - Preliminary activities

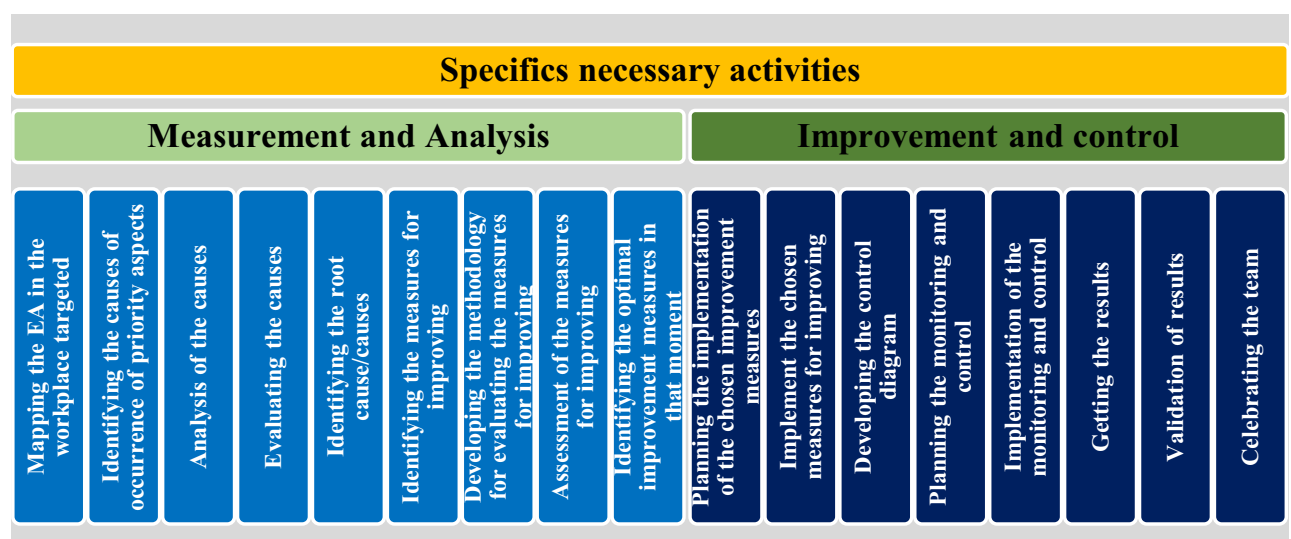

Fig. 2. Phases of the propose DMAIC model - Specifics necessary activities

For implementing the proposed model, the authors took into account the main activities in an agro-touristic structures, summarized in Table 1.

Table 1. Main processes in an agro-touristic structure

\begin{tabular}{|c|c|c|c|c|c|c|}
\hline \multicolumn{2}{|l|}{ Process } & \multicolumn{2}{|l|}{ Included activities } & \multirow{2}{*}{ Input } & \multirow{2}{*}{ Output } & \multirow[b]{2}{*}{ Code } \\
\hline \begin{tabular}{|l|} 
Name \\
\end{tabular} & Code & Name & Code & & & \\
\hline \multirow{4}{*}{ Transport } & \multirow{4}{*}{$\mathrm{T}$} & Gas supply & gs & Gas & Fuelled automobile & TGF \\
\hline & & Carrying out documents & cd & Documents & Documents completed & TDD \\
\hline & & Transport & $\operatorname{tr}$ & Gas & \begin{tabular}{|l|} 
Transportation \\
\end{tabular} & TGT \\
\hline & & Maintenance & me & $\begin{array}{c}\text { Documents, Inspect. } \\
\text { equipment }\end{array}$ & $\begin{array}{c}\text { Automobile } \\
\text { maintained }\end{array}$ & TIA \\
\hline \multirow[t]{2}{*}{ Check in/out } & \multirow[t]{2}{*}{$\mathrm{C}$} & Carrying out documents & cd & $\begin{array}{l}\text { Personal documents. } \\
\text { Specific formulation }\end{array}$ & Check in/out & CPC \\
\hline & & Delivery keys & $\mathbf{d k}$ & Keys & Check in/out & CKC \\
\hline Accommodation & A & Takeover room & $\operatorname{tr}$ & Check in & Check out & $\mathrm{ACC}$ \\
\hline \multirow{4}{*}{ Meal } & \multirow{4}{*}{ M } & Meal preparation & $\mathbf{m p}$ & $\begin{array}{l}\text { Equipment and } \\
\text { products }\end{array}$ & Food prepared & MEF \\
\hline & & Serving food & sf & Meal prepared & Meal served & MPS \\
\hline & & Cleaning table & $\mathbf{d m}$ & Disposal meals & Release tables & MDR \\
\hline & & Cleaning dishes & $\mathbf{c m}$ & Dirty dishes & Clean dishes & $\mathrm{MDC}$ \\
\hline \multirow{2}{*}{$\begin{array}{l}\text { Leisure } \\
\text { activities }\end{array}$} & \multirow{2}{*}{$\mathrm{L}$} & Hike & hk & Transport & Visit & LTV \\
\hline & & Equipment & ha & Equipment & Household cared & LEH \\
\hline \multirow{2}{*}{$\begin{array}{l}\text { Hygiene/ } \\
\text { cleaning }\end{array}$} & \multirow{2}{*}{$\mathrm{H}$} & Common space & cs & $\begin{array}{l}\text { Equipment and } \\
\text { cleaning solutions }\end{array}$ & $\begin{array}{c}\text { Common space } \\
\text { cleaned }\end{array}$ & HEC \\
\hline & & Kitchen and bathrooms & kb & $\begin{array}{l}\text { Equipment and } \\
\text { cleaning solutions }\end{array}$ & $\begin{array}{c}\text { Kitchen and bathrooms } \\
\text { cleaned }\end{array}$ & HEK \\
\hline
\end{tabular}




\begin{tabular}{|c|c|c|c|c|c|c|}
\hline \multicolumn{2}{|l|}{ Process } & \multicolumn{2}{|c|}{ Included activities } & \multirow{2}{*}{ Input } & \multirow{2}{*}{ Output } & \multirow{2}{*}{ Code } \\
\hline Name & Code & Name & Code & & & \\
\hline & & Guest rooms & gr & $\begin{array}{l}\text { Equipment and } \\
\text { cleaning solutions }\end{array}$ & Guest rooms cleaned & HEG \\
\hline & & Household & hh & $\begin{array}{c}\text { Equipment and } \\
\text { cleaning solutions }\end{array}$ & Household cleaned & $\mathrm{HEH}$ \\
\hline \multirow{4}{*}{$\begin{array}{l}\text { Acquisition/ } \\
\text { purchases }\end{array}$} & \multirow{4}{*}{$\mathrm{P}$} & $\begin{array}{c}\text { Analysis and drafting } \\
\text { documents }\end{array}$ & ad & Documents & Documents completed & PDD \\
\hline & & Transport & tt & Gas & \begin{tabular}{|l} 
Transportation \\
\end{tabular} & PGD \\
\hline & & Reception & re & Documents & $\begin{array}{c}\text { Documents completed, } \\
\text { Product reception }\end{array}$ & PDP \\
\hline & & Storage & st & Documents & Storage products & PDS \\
\hline \multirow{3}{*}{$\begin{array}{l}\text { Maintenance } \\
\text { work }\end{array}$} & \multirow{3}{*}{ W } & Maintenance equipment & me & $\begin{array}{l}\text { Documents, } \\
\text { Inspection } \\
\text { equipment }\end{array}$ & Equipment maintained & MDE \\
\hline & & Automobile & at & $\begin{array}{l}\text { Documents, } \\
\text { Inspection } \\
\text { equipment }\end{array}$ & $\begin{array}{l}\text { Automobile } \\
\text { maintained }\end{array}$ & MDA \\
\hline & & $\begin{array}{c}\text { Equipment } \\
\text { and installations } \\
\text { household }\end{array}$ & ei & $\begin{array}{l}\text { Documents, } \\
\text { Inspection } \\
\text { equipment }\end{array}$ & $\begin{array}{c}\text { Equipment } \\
\text { and installations } \\
\text { maintained }\end{array}$ & MDI \\
\hline \multirow{2}{*}{$\begin{array}{l}\text { Agriculture/ } \\
\text { farming }\end{array}$} & \multirow[b]{2}{*}{$\mathrm{F}$} & Mechanized works & mw & Specific equipment & Tillage & FST \\
\hline & & $\begin{array}{c}\text { Manual/handiwork } \\
\text { works }\end{array}$ & $\mathbf{m h}$ & Specific equipment & Tillage & FSH \\
\hline \multirow{3}{*}{ Zootechnics } & \multirow{3}{*}{ Z } & Caring & ca & Specific equipment & Groomed animals & ZSG \\
\hline & & Feeding & fe & Specific equipment & Fed up animals & ZSF \\
\hline & & Slaughtering & sl & Specific equipment & Meat, fur & ZSM \\
\hline \multirow{3}{*}{ Pomiculture } & \multirow{3}{*}{$\mathrm{P}$} & Other manual works & om & Specific equipment & Fruit growing & PSF \\
\hline & & Spraying & sp & Specific equipment & Splash trees & PSS \\
\hline & & Pruning work & pw & Specific equipment & Fruit growing & PSP \\
\hline
\end{tabular}

To achieve the objective of this research, the authors, firstly, focused on identifying the main environmental aspects and impacts associated with them. In this way, the data about the identification and analysis of the aspects are highlighted in Table 2.

Table 2. Identification of environmental aspects and their impacts assessment

\begin{tabular}{|c|c|c|c|c|c|c|c|c|c|c|c|c|}
\hline \multirow[t]{2}{*}{ Process } & \multirow{2}{*}{$\begin{array}{l}\text { Included } \\
\text { activities }\end{array}$} & \multirow{2}{*}{$\begin{array}{l}\text { Input/ } \\
\text { Output }\end{array}$} & \multirow{2}{*}{ Environment aspect } & \multirow{2}{*}{ OC } & \multirow[t]{2}{*}{ EI } & \multicolumn{5}{|c|}{ Impact assessment } & \multicolumn{2}{|c|}{$\begin{array}{c}\text { Impact } \\
\text { classification }\end{array}$} \\
\hline & & & & & & $\mathbf{L}$ & $\mathbf{F}$ & $\mathbf{N}$ & $\mathbf{C}$ & TS & I & $\mathbf{S}$ \\
\hline \multirow{4}{*}{$\mathrm{T}$} & gs & TGF & Exhaust emissions & $\mathrm{N}$ & $\mathrm{AP}$ & 5 & 3 & 1 & 3 & 45 & I & - \\
\hline & cd & TDD & Waste paper & $\mathrm{N}$ & SP & 3 & 5 & 1 & 1 & 15 & $\mathbf{I}$ & - \\
\hline & tr & TGT & Exhaust emissions & $\mathrm{N}$ & $\mathrm{AP}$ & 3 & 3 & 1 & 3 & 27 & I & - \\
\hline & me & TIA & Waste paper & $\mathrm{N}$ & SP & 3 & 1 & 3 & 3 & 27 & I & - \\
\hline \multirow{2}{*}{$\mathrm{C}$} & cd & $\mathrm{CPC}$ & Waste paper & $\mathrm{N}$ & $\mathrm{AP}, \mathrm{SP}$ & 3 & 5 & 1 & 3 & 45 & I & - \\
\hline & dk & $\mathrm{CKC}$ & Waste paper & $\mathrm{N}$ & $\mathrm{AP}, \mathrm{SP}$ & 3 & 5 & 1 & 3 & 45 & I & - \\
\hline A & tr & $\mathrm{ACC}$ & $\begin{array}{c}\text { Waste paper, organic } \\
\text { paper }\end{array}$ & $\mathrm{N}$ & $\mathrm{AP}, \mathrm{SP}$ & 1 & 5 & 3 & 3 & 45 & I & - \\
\hline \multirow{4}{*}{ M } & mp & MEF & Organic waste & $\mathrm{N}$ & SP, WP & 3 & 5 & 1 & 5 & 75 & - & $\mathbf{S}$ \\
\hline & sf & MPS & Organic waste & $\mathrm{N}$ & SP, WP & 3 & 3 & 3 & 1 & 27 & I & - \\
\hline & $\mathrm{dm}$ & MDR & Organic waste & $\mathrm{N}$ & SP, WP & 3 & 3 & 3 & 1 & 27 & I & - \\
\hline & $\mathbf{c m}$ & MDC & $\begin{array}{l}\text { Organic, chemical } \\
\text { waste }\end{array}$ & $\mathrm{N}$ & \begin{tabular}{|c|} 
AP, SP, \\
WP
\end{tabular} & 3 & 3 & 1 & 3 & 27 & $\mathbf{I}$ & - \\
\hline \multirow{2}{*}{$\mathrm{L}$} & hk & LTV & Exhaust emissions & $\mathrm{N}$ & AP & 5 & 3 & 3 & 1 & 45 & $\mathbf{I}$ & - \\
\hline & ha & LEH & Organic, paper waste & $\mathrm{N}$ & SP, WP & 5 & 3 & 1 & 1 & 15 & I & - \\
\hline \multirow{2}{*}{$\mathrm{H}$} & cs & HEC & Chemical waste & $\mathrm{N}$ & $\begin{array}{c}\mathrm{AP}, \mathrm{SP} \\
\mathrm{W}\end{array}$ & 1 & 3 & 5 & 3 & 45 & I & - \\
\hline & $\mathbf{k b}$ & HEK & Chemical waste & $\mathrm{N}$ & \begin{tabular}{|c|} 
AP, SP, \\
WP
\end{tabular} & 1 & 3 & 5 & 3 & 45 & I & - \\
\hline
\end{tabular}




\begin{tabular}{|c|c|c|c|c|c|c|c|c|c|c|c|c|}
\hline \multirow[t]{4}{*}{ Process } & \multirow{2}{*}{$\begin{array}{l}\text { Included } \\
\text { activities }\end{array}$} & \multirow{2}{*}{$\begin{array}{l}\text { Input/ } \\
\text { Output }\end{array}$} & \multirow{2}{*}{ Environment aspect } & \multirow[t]{2}{*}{ OC } & \multirow[t]{2}{*}{ EI } & \multicolumn{5}{|c|}{ Impact assessment } & \multicolumn{2}{|c|}{$\begin{array}{c}\text { Impact } \\
\text { classification }\end{array}$} \\
\hline & & & & & & $\mathbf{L}$ & $\mathbf{F}$ & $\mathbf{N}$ & C & TS & I & $\mathbf{S}$ \\
\hline & gr & HEG & Chemical waste & $\mathrm{N}$ & $\begin{array}{c}\text { AP, SP, } \\
\text { WP }\end{array}$ & 1 & 3 & 5 & 3 & 45 & I & - \\
\hline & hh & $\mathrm{HEH}$ & $\begin{array}{l}\text { Organic, chemical } \\
\text { waste }\end{array}$ & $\mathrm{N}$ & $\begin{array}{l}\text { AP, SP, } \\
\text { WP }\end{array}$ & 5 & 3 & 3 & 1 & 45 & I & - \\
\hline \multirow{4}{*}{$\mathrm{P}$} & ad & PDD & Paper waste & $\mathrm{N}$ & SP, WP & 3 & 3 & 1 & 1 & 9 & I & - \\
\hline & tt & PGD & Exhaust emissions & $\mathrm{N}$ & $\begin{array}{c}\text { AP, SP, } \\
\text { WP }\end{array}$ & 3 & 1 & 3 & 3 & 27 & I & - \\
\hline & re & PDP & Paper waste & $\mathrm{N}$ & SP, WP & 3 & 3 & 1 & 1 & 9 & I & - \\
\hline & st & PDS & Organic, Paper waste & $\mathrm{N}$ & SP, WP & 3 & 3 & 1 & 3 & 27 & $I$ & - \\
\hline \multirow[b]{3}{*}{ W } & me & MDE & Paper waste & $\mathrm{N}$ & SP, WP & 3 & 1 & 1 & & 3 & $\mathbf{I}$ & - \\
\hline & at & MDA & Paper waste, & $\mathrm{N}$ & SP, WP & 3 & 1 & 3 & & 9 & I & - \\
\hline & ei & MDI & $\begin{array}{l}\text { Paper waste, } \\
\text { electronics and } \\
\text { electrical waste }\end{array}$ & $\mathrm{N}$ & $\begin{array}{l}\text { AP, SP, } \\
\text { WP }\end{array}$ & 5 & 1 & 3 & & 15 & I & - \\
\hline \multirow{2}{*}{$\mathrm{F}$} & mw & FST & Organic waste & $\mathrm{N}$ & SP, WP & 3 & 3 & 5 & 3 & 135 & - & $\mathbf{S}$ \\
\hline & $\mathbf{m h}$ & FSH & Organic waste & $\mathrm{N}$ & SP, WP & 3 & 3 & 5 & 3 & 135 & - & $\mathbf{S}$ \\
\hline \multirow{3}{*}{ Z } & ca & $\overline{Z S G}$ & Organic waste & $\mathrm{N}$ & SP, WP & 3 & 3 & 1 & 5 & 45 & $\mathbf{I}$ & - \\
\hline & $\mathrm{fe}$ & ZSF & Organic waste & $\mathrm{N}$ & SP, WP & 3 & 3 & 1 & 5 & 45 & $\mathbf{I}$ & - \\
\hline & sl & ZSM & Organic waste & $\mathrm{N}$ & SP, WP & 3 & 3 & 1 & 5 & 45 & $\mathbf{I}$ & - \\
\hline \multirow{3}{*}{$\mathrm{P}$} & om & PSF & Organic waste & $\mathrm{N}$ & SP, WP & 3 & 3 & 1 & 5 & 45 & $\mathbf{I}$ & - \\
\hline & sp & PSS & Organic waste & $\mathrm{N}$ & SP, WP & 3 & 3 & 1 & 5 & 45 & $\mathbf{I}$ & - \\
\hline & pw & PSP & Organic waste & $\mathrm{N}$ & SP, WP & 3 & 5 & 1 & 5 & 75 & - & $\mathbf{S}$ \\
\hline
\end{tabular}

Legend: WP-water pollution; AP-air pollution; SP-sol pollution; OC-Operating condition; EI-Environment impact; L-Existing of the legal requirements and other environmental requirements regulating environmental impact; F-Frequency of occurring environmental impact; N-The nature of the natural resource/the pollutant /the waste to which it relates those impacts; C-Data on the amount of natural resource/pollutant /waste implied by those impacts; I-Insignificant; S-Significant

The values obtained in Table 2 were analysed using Pareto diagram as follows:

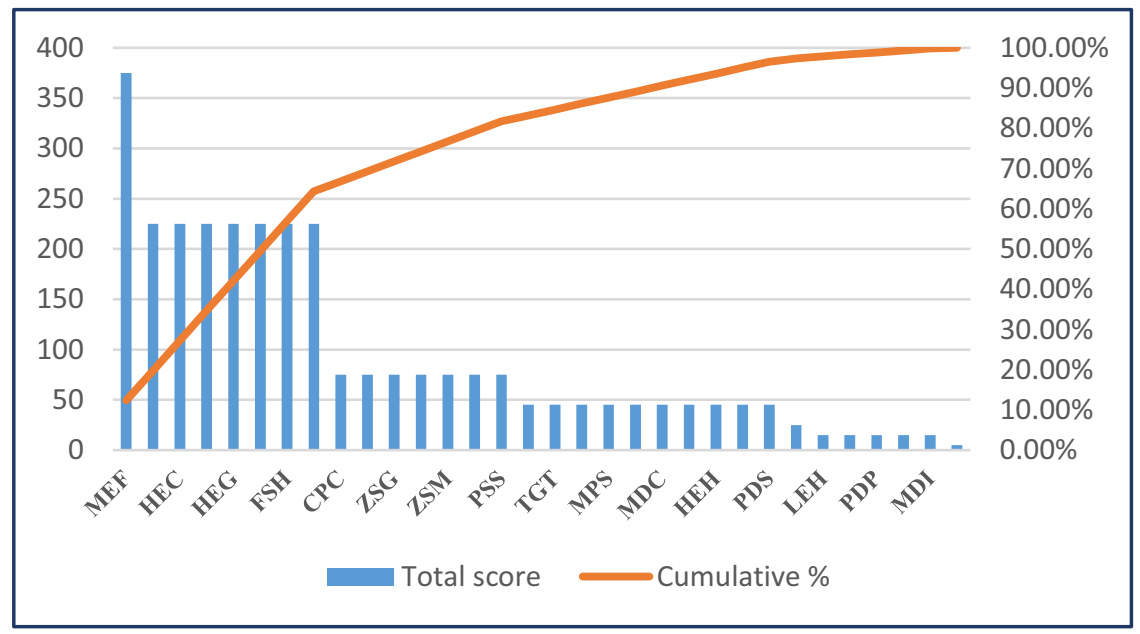

Fig. 4. Overview on analysed values by the instrumentality of Pareto diagram

As can be seen in this chart, $25 \%$ of cases generate about $70 \%$ of the problem. To illustrate how these cases are approached further, below we present the analysis of the first three causes. Therefore, were identified alternative amelioration presented in Table 3. 
Table 3. Identification of alternatives for the top three causes

\begin{tabular}{|c|c|c|c|}
\hline Cause & Alternative 1 & Alternative 2 & Alternative 3 \\
\hline $\begin{array}{c}\text { MFE } \\
\text { Meal preparation } \\
\text { Equipment and } \\
\text { products } \\
\text { Food prepared } \\
\end{array}$ & $\begin{array}{l}\text { Outsourcing } \\
\text { services through } \\
\text { catering }\end{array}$ & $\begin{array}{l}\text { Removal of the meal } \\
\text { by providing the } \\
\text { kitchen. The tourist } \\
\text { will prepare his own } \\
\text { meal }\end{array}$ & $\begin{array}{l}\text { Increasing the consumption } \\
\text { of their own products by } \\
\text { prohibiting the consumption } \\
\text { of products from outside the } \\
\text { agro-touristic structure }\end{array}$ \\
\hline $\begin{array}{c}\mathrm{ACC} \\
\text { Takeover room } \\
\text { Check in } \\
\text { Check out }\end{array}$ & $\begin{array}{l}\text { Creating a special } \\
\text { zone for selective } \\
\text { collection of } \\
\text { waste in agro- } \\
\text { touristic structures }\end{array}$ & $\begin{array}{c}\text { Creating a special } \\
\text { zone, serving the meal } \\
\text { in agro-touristic } \\
\text { structures }\end{array}$ & $\begin{array}{c}\text { Optimizing water } \\
\text { consumption and electricity } \\
\text { by implementing sensors for } \\
\text { light and water }\end{array}$ \\
\hline $\begin{array}{c}\text { HEC } \\
\text { Equipment and } \\
\text { cleaning solutions } \\
\text { Common space } \\
\text { cleaned }\end{array}$ & $\begin{array}{l}\text { Acquisition of eco } \\
\text { cleaning products }\end{array}$ & $\begin{array}{l}\text { Some alert rules for } \\
\text { dirty clothes through } \\
\text { posters with } \\
\text { environmental } \\
\text { protection }\end{array}$ & $\begin{array}{l}\text { Outsourcing services } \\
\text { through specialized firms. } \\
\text { Traditional cleaning }\end{array}$ \\
\hline
\end{tabular}

The alternatives identified will be evaluated using matrix for selecting the alternatives. To apply this tool is necessary to define a number of criteria as below.

The evaluation criteria previously developed alternatives are: a. total cost; b. impact on the problem; c. relation The cost / benefits; d. resistance / impact to change; e. time of implementation; f. the uncertainty about the effectiveness.

To evaluate alternatives to improve relative to these criteria the authors used as an instrument of quality matrix (Table 3 ) for selecting the alternatives where used the following notations: 3 - very favourable impact; 2 - medium favourable impact; 1 - weak favourable impact.

Table 4. The matrix for selecting the alternatives

\begin{tabular}{|c|c|c|c|c|c|c|c|c|}
\hline \multirow[t]{2}{*}{ Cause } & \multirow[t]{2}{*}{ Alternative for improving } & \multicolumn{6}{|c|}{ Criteria selection } & \multirow[t]{2}{*}{ Total } \\
\hline & & $\mathbf{a}$ & $\mathbf{b}$ & $\mathbf{c}$ & d & \begin{tabular}{l|l}
$\mathbf{e}$ & \\
\end{tabular} & f & \\
\hline \multirow{3}{*}{$\begin{array}{c}\text { MFE } \\
\text { Meal } \\
\text { preparation } \\
\text { Equipment } \\
\text { and products } \\
\text { Food prepared }\end{array}$} & Outsourcing services through catering & 2 & 3 & 2 & 2 & 3 & 1 & 14 \\
\hline & $\begin{array}{l}\text { Removal of the meal by providing the kitchen } \\
\text { The tourist will prepare his own meal }\end{array}$ & 3 & 1 & 2 & 2 & 2 & 3 & 13 \\
\hline & $\begin{array}{l}\text { Increasing the consumption of their own } \\
\text { products by prohibiting the consumption } \\
\text { of products from outside the agro-touristic } \\
\text { structure }\end{array}$ & 2 & 3 & 2 & 3 & 3 & 2 & 15 \\
\hline \multirow{3}{*}{$\begin{array}{c}\text { ACC } \\
\text { Takeover } \\
\text { room } \\
\text { Check in } \\
\text { Check out }\end{array}$} & $\begin{array}{c}\text { Creating a special zone for selective } \\
\text { collection of waste in agro-touristic structures }\end{array}$ & 2 & 3 & 2 & 2 & 3 & 1 & 14 \\
\hline & $\begin{array}{l}\text { Controlling serving the meal in the room by } \\
\text { creating a special zone }\end{array}$ & 2 & 2 & 3 & 2 & 2 & 1 & 12 \\
\hline & $\begin{array}{l}\text { Optimizing water consumption and } \\
\text { electricity by implementing sensors for } \\
\text { light and water }\end{array}$ & 1 & 3 & 3 & 3 & 2 & 1 & 13 \\
\hline \multirow{3}{*}{$\begin{array}{c}\text { HEC } \\
\text { Equipment } \\
\text { and cleaning } \\
\text { solutions } \\
\text { Common } \\
\text { space cleaned }\end{array}$} & Acquisition of eco cleaning products & 1 & 3 & 2 & 3 & 2 & 1 & 12 \\
\hline & $\begin{array}{l}\text { Some alert rules for dirty clothes through } \\
\text { posters with environmental protection }\end{array}$ & 3 & 2 & 3 & 3 & 2 & 2 & 15 \\
\hline & $\begin{array}{c}\text { Outsourcing services through specialized } \\
\text { firms }\end{array}$ & 2 & 3 & 2 & 3 & 2 & 1 & 13 \\
\hline
\end{tabular}


After identifying suitable options, using matrix for selecting the alternatives, a plan of measures will be developed to improve compliance with the requirements of Table 5 .

Table 5. Plan of measures to improve compliance

\begin{tabular}{|c|c|c|c|c|c|}
\hline No. & Cause & Corrective action & Resources needed & Responsible & Term \\
\hline 1. & $\begin{array}{c}\text { MFE } \\
\text { Meal } \\
\text { preparation } \\
\text { Equipment } \\
\text { and products } \\
\text { Food } \\
\text { prepared }\end{array}$ & $\begin{array}{l}\text { Increasing the } \\
\text { consumption of } \\
\text { their own products } \\
\text { by prohibiting the } \\
\text { consumption of } \\
\text { products from } \\
\text { outside the agro- } \\
\text { touristic structure }\end{array}$ & $\begin{array}{c}\text { M: preparing from } \\
\text { the own products } \\
\text { U: The chef }\end{array}$ & The owner & Daily \\
\hline 2. & $\begin{array}{c}\text { ACC } \\
\text { Takeover } \\
\text { room } \\
\text { Check in } \\
\text { Check out }\end{array}$ & $\begin{array}{l}\text { Optimizing water } \\
\text { consumption and } \\
\text { electricity by } \\
\text { implementing } \\
\text { sensors for light and } \\
\text { water }\end{array}$ & $\begin{array}{l}\text { M: sensors for } \\
\text { light and water } \\
\text { U: the electrician, } \\
\text { the plumber }\end{array}$ & The owner & $\begin{array}{c}2 \\
\text { month }\end{array}$ \\
\hline 3. & $\begin{array}{c}\text { HEC } \\
\text { Equipment } \\
\text { and cleaning } \\
\text { solutions } \\
\text { Common } \\
\text { space cleaned }\end{array}$ & $\begin{array}{l}\text { Some alert rules for } \\
\text { dirty clothes } \\
\text { through posters with } \\
\text { environmental } \\
\text { protection }\end{array}$ & $\begin{array}{l}\text { M: posters with } \\
\text { environmental } \\
\text { protection } \\
\text { U: the staff }\end{array}$ & The owner & 1 day \\
\hline
\end{tabular}

Legend: $\mathrm{M}$ - material resources, $\mathrm{U}$ - humane resource

With the control elements we have to ensure that corrective actions planned are used and maintained as required in Table 6 , highlighting those items graphed control.

Table 6. Corrective actions planned

\begin{tabular}{|c|c|c|c|}
\hline Variable & $\begin{array}{l}\text { Increasing the consumption } \\
\text { of their own products by } \\
\text { prohibiting the } \\
\text { consumption of products } \\
\text { from outside the agro- } \\
\text { touristic structure }\end{array}$ & $\begin{array}{l}\text { Optimizing water } \\
\text { consumption and } \\
\text { electricity by } \\
\text { implementing sensors for } \\
\text { light and water }\end{array}$ & $\begin{array}{l}\text { Some alert rules } \\
\text { for dirty clothes } \\
\text { through posters } \\
\text { with } \\
\text { environmental } \\
\text { protection } \\
\end{array}$ \\
\hline $\begin{array}{l}\text { How to } \\
\text { measure }\end{array}$ & Tracking stock & Tracking consumption & Visual \\
\hline Were & Storage area & $\begin{array}{l}\text { At the electric and water } \\
\text { counter }\end{array}$ & Rooms \\
\hline Reference & Sheet Storage & The bill & $\begin{array}{l}\text { The amount of } \\
\text { washing }\end{array}$ \\
\hline $\begin{array}{c}\text { Who } \\
\text { measures }\end{array}$ & The chef & The owner & The staff \\
\hline Who decides & The chef & The owner & The owner \\
\hline What makes & Remake stock & Checking the sensors & $\begin{array}{l}\text { Checking the } \\
\text { posters }\end{array}$ \\
\hline Who checks & The owner & The owner & The staff \\
\hline Frequency & Dailey & Monthly & Dailey \\
\hline Were records & Register & Register & Register \\
\hline
\end{tabular}




\section{Conclusions}

In order to comply with the international environment requirements, the authors recommend that SMEs in the agro-tourism sector also, to assume the techniques, tools and modern methods of control, monitoring and improvement of environmental aspects. In this context the authors presented in the present paper a conceptual model that is meant to facilitate the purpose defined in the paper title.

Currently, there are numerous studies, research and methodologies that consecrate optimally chosen can contribute to achieving specific environmental objectives. For example, to establish the strategy to improve the waste can use other tools and methodologies from other fields. In this paper the authors attempted to clarify the relationship between the agrotourism activities and environment. The extent to which succeeded this, it is up to the potential users of the model [27].

\section{References}

1. $* * *$ http://eurlex.europa.eu/summary/chapter/environment.html?root_default= SUM_1_CODED $=20 \&$ locale $=$ en, accessed 6.03.2017

2. M. Schiavon, M. Redivo, G. Antonacci, E.C. Rada, M. Ragazzi, D. Zardi, L. Giovannini, Athmos. Environ. 120 (2015)

3. E.C. Rada, WIT Transact. Ecol. Environ. 191 (2014)

4. E.C. Rada, C. Zatelli, P. Mattolin, WIT Transact. Ecol. Environ. 180 (2014)

5. R.D. Ionescu, M. Ragazzi, L. Battisti, E.C. Rada, G. Ionescu, WIT Transact. Ecol. Environ. 176 (2013)

6. European Union, https://europa.eu/european-union/about-eu/history/1970-1979_en, accessed 6.03.2017

7. $* * * h t t p: / / e u r-l e x . e u r o p a . e u / l e g a l-c o n t e n t / E N / T X T / ? u r i=u r i s e r v \% 3 A x y 0026$, accessed 04.03.2017

8. European Commission, http://ec.europa.eu/environment/action-programme/, accessed 04.03.2017

9. $* * * \mathrm{http}: / /$ legislatie.just.ro/Public/DetaliiDocumentAfis/1725, accessed 04.03.2017

10. ${ }^{* * *} \mathrm{http}: / /$ www.gppbest.eu/?page id=46\&lang=ro, accessed 04.03.2017

11. European Union, Consolidated Versions of the Treaty on European Union and the Treaty on the Functioning of the European Union, Charter of Fundamental Rights of the European Union (Publications Office of the European Union, p. 132, 2010)

12. ***, https://www.iso.org/iso-14001-environmental-management.html, accessed 04.03.2017

13. European Commission, http://ec.europa.eu/environment/emas/index_en.htm, accessed 04.03.2017

14. European Parliament, M. Juul, EPRSTourism and European Union - Recent trends and policy developments (European Parliament, 2015)

15. P. Sharon, C. Hunter, K. Blackstock, Tourism Manag. 31, 6 (2008)

16. M. Cawley, D.A. Gillmor, Annals of Tourism Res. 35, 2 (2008)

17. D. Hall, M. Mitchell, L. Roberts, Tourism and the countryside: Dynamic relationships, In: Hall D, Mitchell M, Roberts L, editors. New Directions in Rural Tourism (Aldershot, 2003)

18. I. Frochot, Tourism Manag. 26, 3 (2005)

19. European Parliament, http://www.europarl.europa.eu/thinktank/en/document.html?reference=EPRS_BRI(2016)58 1978 , accessed 04.03.2017

20. N. G. McGehee, K. Kyungmi, G.R. Jennings, Tourism Manag., 28, 1 (2007)

21. C. Tew, C. Barbieri, Tourism Manag. 33, 1 (2012)

22. N. Polovitz Nickerson, R. J. Black, S. F. McCool, J. Travel. Res. 40, 1 (2001)

23. G. Veeck, D. Che, A. Veeck, The Professional Geograph. 58, 3 (2006) 
24. L. Mastronardi, V. Giaccio, A. Giannelli, A. Scardera, https://agriregionieuropa.univpm.it/ it/content/article/31/40/ agriturismo-e-sostenibilita-ambientale-primi-risultati-di-unanalisiaziendale, accessed 6.03.2017

25. C.V. Kifor, C. Oprean, Ingineria Calității. Imbunatațirea 6 Sigma (Editura Universitatii "Lucian Blaga" din Sibiu, 2006)

26. M.V. Zerbes, L.I. Cioca, "Quality - Access to Success", 1, S1 (2017)

27. S. Bungau, R. Suciu, A. Bumbu, G. Cioca, D.M. Tit, J. Environ. Protect. Ecol. 16, 3 (2015) 